Sadio-Ramos, F.J., Ortiz-Molina, M.A., \& Bernabé Villodre, M.M. (2020). La formación del profesorado de Música para potenciar la creatividad desde la utilización de las TIC: una experiencia biográfica. Revista Electrónica Interuniversitaria de Formación del Profesorado, 23(2), 155-166.

DOI: https://doi.org/10.6018/reifop.422891

\title{
La formación del profesorado de Música para potenciar la creatividad desde la utilización de las TIC: una experiencia biográfica
}

Fernando José Sadio-Ramos', María Angustias Ortiz-Molina², María del Mar Bernabé Villodre ${ }^{3}$

${ }^{1}$ Politécnico de Coimbra, Escola Superior de Educação; ${ }^{2}$ Universidad de Granada, Grupo de Investigación HUM-672 AREA; ${ }^{3}$ Universidad de Valencia

\section{Resumen}

Presentamos parte de una investigación cualitativa de tenor biográfico-narrativo; su principal objetivo es recoger experiencias de formación de profesores/ investigadores de enseñanza superior que utilicen las TIC como un instrumento importante de formación, contribuyendo al desarrollo de las competencias de creatividad, colaboración, cooperación y comunicación, necesarias en una educación integral volcada para la sostenibilidad curricular y sus implicaciones en una ciudadanía participativa. Aquí, tratamos de la presencia de las TIC en la formación del profesorado en materia musical y como esta inclusión puede llevar al desarrollo de la creatividad mediante actividades cooperativas, como verificamos analizando el relato de experiencia y práctica docente e investigadora de Elena Riaño, en señalada coincidencia con las aportaciones teóricas y empíricas referidas.

\section{Palabras clave}

Creatividad; Educación Musical; Investigación biográfico-narrativa; Profesorado; TIC.

\section{Contacto:}

Fernando José Sadio-Ramos, framos@esec.pt, R. Dom João III, s/n, Solum, 3030-329 Coimbra Portugal. 


\title{
Music teachers' Training to enhance creativity from the use of ICT: a biographical experience
}

\begin{abstract}
The paper is part of an investigation that uses qualitative biographical-narrative research procedures. Its main objective is to collect training experiences of Higher Education teachers and researchers in which the use of ICT is an important training tool, seen as contributing to the development of creativity, collaboration, cooperation and communication skills, in a holistic education perspective focused on curricular sustainability and its implications in terms of participatory citizenship. Here, it is studied the presence of ICT in the training of musical education teachers and how its inclusion can lead to the development of creativity through cooperative activities by analyzing Elena Riaño's teaching and research practice, in relevant coincidence with the referred theoretical and empirical contributions.
\end{abstract}

\section{Key words}

Bio-narrative Research; Creativity; Musical Education; ICT; Teachers.

\section{Introducción}

El Espacio Europeo de Educación Superior (EEES) llevó a las universidades a destacar las conexiones entre contenidos teóricos y prácticos, lo que vendría a traducirse en un "saber hacer" y en el desarrollo de competencias consideradas básicas (Galera y Mendoza, 2011), como:

(1) Comunicación oral.

(2) Trabajo en equipo: cooperatividad y colaboratividad.

(3) Creatividad.

El EEES trajo igualmente el hecho de que el alumnado debía "aprender a aprender", traducido en trabajar en el aula la capacidad para gestionar y compartir información. Esto puede venir de la mano de nuevas formas de comprender el proceso de enseñanza/aprendizaje como las creadas por las TIC (Alonso, Muñoz y Quiñones, 2018), facilitadoras de la enseñanza constructivista (Casanova y Serrano, 2016). No puede obviarse que las TIC son una realidad en el ámbito universitario (Roldán, 2016) que llegaron a cambiar la forma de entender la educación (Colás-Bravo y Hernández-Portero, 2017), cambiándose el paradigma educativo al entrar el modelo tradicional en crisis (Casanova y Serrano, 2016); incluso, Marqués (2004) ha señalado como las TIC se convirtieron en un eje transversal para la formación, a distintos niveles educativos, generando oportunidades para recrear y solucionar problemas de la vida actual (Rodríguez y Guzmán de Castro, 2012). Cózar, De Moya, Hernández y Hernández (2015) llegan a considerar las TIC como "incuestionables señas identitarias de toda una generación" (p. 73). Estos argumentos llevan a considerar las TIC como posibilitadoras de un valioso cambio en el proceso de enseñanza/aprendizaje mediante un nuevo soporte (Lopes y Gomes, 2018). 
Esta presencia de las TIC, que ha derivado en las situaciones de enseñanza/aprendizaje comentadas, se convirtió en un reto para el profesorado al tener que adaptar su práctica docente (Colás-Bravo y Hernández-Portero, 2017), utilizando materiales digitales habilitadores de distintos tipos de apropiación de los contenidos disciplinares (Caramés, 2019). Así, la Educación Superior debe formar profesionales con saberes, habilidades, destrezas y herramientas que les permitan mejorar los procesos de información y comunicación mediante, entre otros, el trabajo en equipo (Alonso, Muñoz y Quiñones, 2018) y las competencias digitales para introducir las tecnologías en la práctica diaria (ColásBravo y Hernández-Portero, 2017); no obstante, referimos que Palau, Usart y Ucar (2019) han señalado que la comprensión de la competencia digital no vendría solo conformada por la adquisición de un conjunto de destrezas tecnológicas, sino que hablaríamos de la necesidad de relacionar la competencia digital con las restantes competencias curriculares (Escudero, Martínez-Domínguez y Nieto, 2018).

Ante esto, surgiría la necesidad de la incorporación de las TIC durante la formación universitaria, promoviendo un uso educativo de la tecnología desde las materias curriculares del Grado (Castañeda, 2019) y así dar respuesta a las necesidades formativas del alumnado actual, que demanda actividades motivadoras, activas y con herramientas tecnológicas cercanas (tabletas, teléfonos, etc.), puesto que las tecnologías cada vez resultan más imprescindibles en educación, como "eco" de los cambios de la sociedad actual (Díez, 2018).

Todo esto nos lleva a concretar el área de estudio comprendida en esta investigación: la presencia de las TIC en la formación del profesorado, concretamente, en materia musical y que esta inclusión puede llevar al desarrollo de la creatividad mediante actividades cooperativas. Touriñán (2018) consideró que era posible integrar las TIC en la docencia musical en el entorno universitario para potenciar y facilitar los aprendizajes; otros autores llegaron a conclusiones parecidas, incidiendo en lo deficitario de la formación en TIC del futuro profesorado de música (Díez, 2018) o en la necesidad de formación en software libre musical (Vaquer y Vera-Muñoz, 2012).

\section{Marco teórico}

\section{La presencia de las TIC en la formación del profesorado de Música}

La inclusión de las TIC en el currículo musical de Educación Primaria/ Secundaria puede considerarse una realidad, dada la gran cantidad de experiencias didácticas relacionadas (Castañón y Vivaracho, 2012; Vargas, Gértrudix y Gértrudix, 2015; Padula, 2016; Espigares y Bautista, 2018); Cózar, De Moya, Hernández y Hernández (2015) afirman en este mismo sentido que la difusión y el consumo musical están fuertemente asociados a su desarrollo. Todavía, ¿cuánto se ha investigado sobre la formación en TIC desde las materias musicales propias del plan de estudios del futuro profesorado de Música? Sin experiencias innovadoras durante la formación universitaria, no podrán realizarse experiencias innovadoras en las etapas de Primaria/ Secundaria desde las TIC, máxime cuando se asocia a las TIC un enorme potencial para la enseñanza musical (Colás-Bravo y Hernández-Portero, 2014).

Así, Galera y Mendoza (2011) realizaron una experiencia durante la formación del profesorado de Música de Primaria, según la cual muestran que la inclusión de las TIC facilita el aprendizaje y el desarrollo de la capacidad creativa, al tiempo que posibilita el fomento de conductas cooperativas. Díez (2018) señaló en sus investigaciones que la falta de formación del profesorado de Música puede llevar a la escasa y desigual incorporación 
de las TIC en los centros educativos, de ahí la necesidad de insistir en la realización de experiencias similares a las ya comentadas. Cózar, De Moya, Hernández y Hernández (2015) investigaron las TIC deben entenderse siendo herramientas con un importante poder didáctico, a partir de su profundización en la competencia musical respecto a ellas. PalazónHerrera (2016) ha considerado que esa incorporación de las TIC al ámbito educativoformativo musical debería partir de su interpretación de recurso de apoyo al aprendizaje, atendiendo al posible uso otorgado por el profesorado durante las etapas de Primaria/ Secundaria. Lores, Sánchez y García (2019) investigaron sobre la brecha entre formación en competencia digital y la formación que debería recibirse durante la formación del profesorado de Primaria, mostrando que se observa un déficit en la competencia derivado de una oferta digital instrumental y teórica, alejada de las necesidades docentes reales.

Casanova y Serrano (2016) centraron su experiencia investigadora en la formación orientada a garantizar una introducción óptima y crítica de las TIC en la futura enseñanza de la Música en distintas etapas educativas. Touriñán (2018) indicó que la incorporación de las TIC al aula de formación del profesorado de Música también puede suponer el desarrollo de competencias relacionadas con el pensamiento crítico, de gran interés para el futuro alumnado bajo su tutela.

Como señala Hepp (2002), el aumento del interés en combinar educación musical con formación en TIC vendría determinado por: el fácil acceso a materiales y partituras, el fácil uso de los programas para hacer música y la motivación que todo ello supone para el alumnado. A todo esto, que vendría a "facilitar" la utilización de la tecnología en el aula, se suman Vaquer y Vera-Muñoz (2012) que han señalado que el profesorado se muestra interesado en la formación para la utilización de las TIC en el aula musical, recurriendo a cursos de formación continua para complementar su escasa o nula formación en recursos tecnológicos específicos. Díez y Carrera (2018) expusieron también en sus investigaciones esa predisposición hacia la utilización de las TIC. En relación con esto, Colás-Bravo y Hernández-Portero (2017) insistieron en que una adecuada formación permanente podría paliar la escasez de formación tecnológica inicial del profesorado de Música.

Pero, nada de todo lo comentado será posible si el profesorado no cambia sus creencias y su autopercepción sobre sus propias capacidades ante la utilidad de las TIC, que condicionan su uso en el aula (Colás-Bravo y Hernández-Portero, 2014). Deberían comprenderlas, de acuerdo con Cabero y Martínez (2019) siendo herramientas para enriquecer, crear y generar conocimientos.

\section{La creatividad en la formación del profesorado de Música desde/con las TIC}

El ser humano es capaz de producir múltiples y distintas manifestaciones artísticas, que entrarían a formar parte del desarrollo cultural de una sociedad. Esta productividad, se la debe a su capacidad creativa, innata a todas las personas (Lago y Ponce de León, 2012), aunque también puede entrenarse (Vecina, 2006). ¿El infante nace creativo o debe desarrollarse su creatividad mediante distintas herramientas? ¿Como se enfoca la formación del profesorado para poder "extraer" del alumnado todo su potencial creativo? ¿Se prepara al futuro docente para hacer frente a la formación creativa del alumnado? Todos y cada uno de estos interrogantes parten según entendamos la creatividad y, a partir de ahí, su posible relación con las TIC, que iremos detallando seguidamente.

Vecina (2006) definió la creatividad como un ejercicio de libertad donde no existe tanta determinación de los estímulos externos cuanto de los proyectos y metas que la propia 
libertad crea. Por su parte, Torres (2017) consideró la creatividad siendo una capacidad, cualidad o característica que todos poseemos.

La creatividad puede desarrollarse, pero al mismo tiempo resultará de una confluencia de características personales, cognitivas, técnicas, sociales y culturales (Vecina, 2006). Torres (2017) considera que la creatividad no es que pueda desarrollarse, sino que debe desarrollarse y necesita, así, entrenamiento y estimulación. Consecuentemente, será el profesorado quien deba motivar y estimular al alumnado para dicho descubrimiento de sus capacidades creativas; aunque, esto pasaría por haber guiado al futuro profesorado previamente durante su formación.

En este sentido, las experiencias musicales de Galera y Mendoza (2011) muestran que un enfoque metodológico que parta de las TIC puede fomentar actitudes creativas y cooperativas en el aula formativa del futuro docente. Vargas, Gértrudix y Gértrudix (2015) han realizado experiencias compositivas colaborativas desde las TIC, mostrando que este tipo de planteamiento didáctico posibilita una retroalimentación que no se genera con la composición, en su sentido tradicional. Podría hablarse, así, de una mediatización del vínculo creativo debido al desarrollo tecnológico, que ha ofrecido herramientas para ello (Padula, 2016). En esa línea del carácter cooperativo, Acosta, Martín-García y Hernández (2019) han señalado que la mediación de las TIC posibilita aprendizajes colaborativos y ahí, precisamente, estribaría lo necesario de potenciar su uso durante la formación, que favorecería iniciarse en el trabajo cooperativo y colaborativo que terminarán desarrollando al formar parte de equipos docentes en sus futuros centros de trabajo.

Otros autores Martín-Piñol, Calderón-Garrido y Gustems-Carnicer (2016), por ejemplo, han realizado experiencias didácticas desde la formación del profesorado en las que las TIC se convierten en herramienta para ensamblar materias artísticas, cuyo proceso formativo pueda enfocarse desde el aprendizaje de las competencias comunes. Casanova y Serrano (2016) han insistido en que la formación digital y la creatividad de cada docente serán las que marquen el planteamiento de nuevos recursos y posibilidades didácticas para el aula. De todo esto pueden deducirse las necesidades formativas iniciales en TIC del futuro profesorado para que la capacidad creativa no se vea mermada o dificultada, a la hora de plantear recursos para las distintas etapas educativas.

\section{Metodología}

La investigación, de que se presenta aquí uno de sus productos, utiliza procedimientos de investigación cualitativa biográfico-narrativa: las entrevistas en profundidad. Su principal objetivo es recoger experiencias de formación de profesores/ investigadores de enseñanza superior que utilicen las TIC como un instrumento importante de formación, contribuyendo al desarrollo de las competencias de creatividad, colaboración, cooperación y comunicación, necesarias en una educación integral volcada para la sostenibilidad curricular y sus implicaciones en una ciudadanía participativa. El objeto de estudio es constituido por los discursos recogidos y reconstituidos en las entrevistas. El artículo contiene los resultados obtenidos en una entrevista en profundidad hecha a una profesora/ investigadora con un currículo considerable en formación universitaria de profesorado, María Elena Riaño Galán (Universidad de Cantabria), que autorizó la divulgación de su identidad. Efectuada y grabada por Skype, se transcribió la entrevista y fue finalmente validada por Elena.

El instrumento plantea la cuestión ética del anonimato/ confidencialidad y consideraciones biográfico-curriculares del entrevistado. Si respecto al anonimato la informante pretendió la 
divulgación del nombre -la propia visibilidad de los proyectos referidos lo tornaría inútil, probablemente-, ya en cuanto a la grabación bruta y correspondiente texto transcrito decidió mantenerlos confidenciales. El cuidado ético de la investigación se completó firmando la declaración de consentimiento informado. Las preguntas, planteadas en términos temáticos y abiertos, cubren las áreas pertinentes al tratamiento de los aspectos biográficos relevantes para la temática general de investigación.

El análisis textual e inductivo de la entrevista posibilitaron la recogida de las percepciones/ opiniones que presentamos.

\section{Resultados}

\section{Sinopsis biográfica y curricular}

Elena, de 48 años de edad, es detentora del Título Superior de Música en la especialidad de Piano (Real Conservatorio Superior de Música de Madrid) y Doctora en Filosofía y Ciencias de la Educación (Programa de Psico-Didáctica, Universidad del País Vasco). Desarrolla su labor docente e investigadora en la Universidad de Cantabria (Departamento de Educación, Facultad de Educación) desde el año 2000. Esta actividad profesional fue antecedida de trabajo con la música en el Conservatorio, en diferentes centros de Educación Secundaria Obligatoria y en ámbitos educativos no-formales.

Además de esta actividad, su recorrido profesional cuenta con el desarrollo de la faceta intérprete de piano (como solista y acompañante). En la Universidad hace Formación, Investigación y Gestión (fue directora del Aula de Música de la Universidad de Cantabria gestión cultural- y es actualmente subdirectora del Departamento de Educación). Ha sido Miembro del Consejo de Dirección de "Revista Eufonía. Didáctica de la Música”, y Miembro del Consejo de Redacción de "Revista Electrónica Complutense de Investigación en Educación Musical” (Universidad Complutense de Madrid).

Tiene un amplio recorrido de Investigación, participando en diferentes proyectos (con financiación de ámbito privado y fondos públicos), de que refiere algunos (de carácter nacional): (1) 2012, proyecto sobre Patrimonio compartido: «Música y arte ritual en el ámbito iberoamericano» (liderado por Maravillas Díaz, de la Universidad del País Vasco y Pilar Barrio, de la Universidad de Extremadura); (2) actualmente tiene un equipo de trabajo con el que comparte investigación: Adolf Murillo (Universidad de Valencia), Noemy Berbel (Universidad de las Islas Baleares), Cristina Arriaga (de la Universidad del País Vasco), Alberto Cabedo (de la Universidad de Castellón); (3) proyecto aprobado por la Fundación Daniel \& Nina Carasso «Recolectando Arte, Sociedad y Educación a través de las Artes», liderado por Adolf Murillo (Universidad de Valencia).

De este último, destaca que la llevó «a trabajar una visión un poco más interdisciplinar de las artes, de que el origen es la Educación Musical, pero con una noción de hibridación, de ruptura de barreras entre los lenguajes artísticos. Esto nos lleva ya también a trabajar con la realidad social, de lo que ocurre con la realidad».

Desarrolla actualmente un proyecto «I+D del Ministerio (...) liderado por Noemy Berbel y que se titula "Rehabilitar el barrio: proceso de rehabilitación y empoderamiento entre universidad, escuela y sociedad a través de prácticas artísticas"; (...) entramos aquí ya de lleno en un trabajo compartido entre artistas, docentes, familias, el propio alumnado en una idea de comunidad y de práctica».

Del recorrido profesional concluye: «El trabajo con proyectos, el uso de la tecnología la visión interdisciplinar ha sido hacia donde yo me he ido dirigiendo; tanto mis colegas de 
trabajo como yo misma nos hemos ido dirigiendo hacia esta visión un poco caleidoscópica, multidisciplinar y con la generación de proyectos artísticos globales que sean significativos, con entornos que sean reales tengo en cuenta la realidad social contemporánea». La transformación personal holística posibilitada por este trabajo de investigación y docencia es reconocida por la entrevistada, refiriendo que la propia práctica docente y la realidad investigadora la han ido formando como persona y es lo que está realizando ahora mismo como investigadora.

El colofón de cierre de temática reside en la publicación y socialización del saber producido: «Esto te lleva a publicar, a hacer talleres, pero fundamentalmente a publicar que es lo que ahora mismo exige la universidad, iy en eso estamos!»

\section{Las TIC en la investigación y docencia de Elena}

El trabajo de Elena presupone, fundamentalmente, la implicación de sus alumnos, repartida por diferentes planos. En uno, la realización de trabajos de investigación («trabajamos con colegios, con niños de Educación Infantil y de Educación Primaria, incluso con adolescentes»), en otro, con los futuros Maestros (estudiantes de Grado) y Maestros (Máster en Educación Primaria). El interés señalado hace con que su enseñanza repercuta en todos los implicados. En primer lugar, en ella misma, en términos de experiencias acumuladas y de constante autocrítica, destacando su particular cuidado en cuestionar su trabajo y conectarlo con la realidad sociocultural. Entiende que la Educación Artística y la Educación Musical tienen que ir de la mano de la sociedad, la cultura, los avances, y no se puede enseñar de forma aislada encerrada entre cuatro paredes cuando fuera están ocurriendo cosas muy importantes. Eso la hace buscar constantemente modelos creativos e interactivos, en que los estudiantes sean absolutamente los protagonistas, de acuerdo con el cambio preconizado por el EEES; piensa que para «el Plan Bolonia las clases expositivas pasaron a segundo plano, también un examen único lo pasó, la enseñanza teórica también. (... ) con estas reformas se incluyeron metodologías docentes colaborativas, participativas, de trabajo individual y colectivo; el sistema de evaluación también cambió, se pasó a una enseñanza más práctica...». Todo esto potencia «la capacidad autónoma del estudiante en la búsqueda de información, de indagación, unas horas presenciales y otras no, y busca (...) un desarrollo de competencias para su futura labor profesional». En esto «estamos todos»y «lo que no puede ocurrir es no tener en cuenta todo esto»; en consecuencia, cree que «Todo lo que yo haga en estos sentidos repercutirá positivamente en los estudiantes».

En cuanto a sus preocupaciones metodológicas (agrupamiento de alumnos, uso de espacios, estrategias utilizadas, materiales), pone en relieve que trabaja «de manera colaborativa, interdisciplinar y desde luego incluy[e] la tecnología». Refiere que su «interés por la tecnología surge de una manera orgánica, como una necesidad docente» y que no concibe «la tecnología con una visión instrumentalista». Como tal, siempre ha sido una «herramienta más, que permitía a los estudiantes poder generar lo que querían y poderles ayudar en la ejecución y el desarrollo de la idea de lo que querían hacer», esencial para el desarrollo de proyectos vistos como «creaciones originales por los distintos grupos». Así, desde siempre recorrió a «prácticas de registro sonoros, (...) de grabación de audio, de geolocalización -a través de mapas, por ejemplo-; productos audiovisuales tipo documental, cortes de grabación hechos con el móvil, utilizando también el ordenador...», según el proyecto, siempre suponiendo sus «muchas posibilidades creativas», con niños, por ejemplo. "Yo uso las herramientas tecnológicas ..., no es que yo sea una experta en ellas, sino que ayudo o guío a los estudiantes para que cada uno busque la herramienta que necesita con el fin de potenciar esa creatividad que tienen y ayudar en la ejecución de esa 
idea que han imaginado, que han diseñado en el proyecto que estemos revisando». Elena subraya que su formación es de tenor clásico - «yo procedo de una educación clásica, de conservatorio, una formación instrumental»-, pero se abre a la utilización -«muy básica»de la tecnología, que le despierta vértigo por su cambio aceleradísimo, pero que no constituye obstáculo ya que «no necesitamos tampoco grandes cosas».

Elementos tecnológicos utilizados en sus clases: «grabadoras de audio (me interesan las capturas de sonido), cámaras de fotos, cámaras de vídeo para capturas de imágenes, móviles, el móvil siempre lo introduzco (...), micrófono, amplificadores, una mesa de sonido. En cuanto a instrumentos, utilizo los acústicos, algunos amplificados como la guitarra o bajo eléctrico, y todos aquellos que tenga disponibles; (...) trabajamos por supuesto también las posibilidades sonoras de objetos cotidianos, concibiendo así el objeto como instrumento sonoro»; igualmente, «editores de fotos para manipularlas, o editores de vídeo (...) [aunque sean de] los más básicos [como] Audacity, Garage Band o Logic, para audio; o Imovie para video (dependiendo si tienen Mac o PC); el Illustrator a veces lo usan para temas de diseños (un cartel, una entrada para un evento, etc.)». La cooperación es facilitada tecnológicamente: «a veces estamos trabajando un grupo y necesitamos que los 5 o 6 estudiantes estén escuchando un mismo audio y para ello tenemos multiconectores de auriculares con 5 puertos. Esto les permite poder escuchar al mismo tiempo en el mismo equipo».

Su actitud es abierta, de adaptación «al material que tenga, a los espacios de trabajo con los que cuento"; asume el papel de "guía, ayuda a los grupos» que -aunque no sabiendo de todo- coloca a los alumnos en la situación de que «son ellos mismos los que hacen esa labor de indagación y yo voy aprendiendo con ellos, hay una co-creación». Esta función de guíaentendedora parte de la asunción de que las TIC no sustituyen la tecnología anterior, sino que «son una herramienta más, (...) recurso más»; «Tradición e innovación no son compartimentos estancos, (...) podemos reinterpretar un proyecto o hacer uno nuevo basándonos en obras del pasado». Además, los roles de los estudiantes van cambiando con «este trabajo (... ): ilustrador, editor, escritor, fotógrafo, compositor, coreógrafo... ».

Un último punto que destacar en esta concepción estructurante de su posición pedagógica es la importancia que las TIC asumen -sin exclusividad- al «fomentar marcos de trabajo que contribuyan al desarrollo de las habilidades creativas dentro de entornos colaborativos». Acrece a esto «que no repito nunca un proyecto y esto me resulta motivador y sugerente ¿no?, a ver qué proyecto vamos a hacer el próximo curso, ¿no? Es una mirada abierta, es la idea de proceso abierto que no termina, y eso la verdad es que es muy motivante. No sé..., es lo que intento con los estudiantes y es un reto personal y profesional que me planteo curso a curso». Actualmente, se ocupa de la utilización de sensores en los proyectos a implementar.

Elena presenta su satisfacción y realización personal por el hecho de que esta práctica pedagógica genere productos correspondientes. «A mí, todo esto me emociona, ipues si consigues contagiar a los estudiantes, pues ya es algo realmente maravilloso!»

No ve la creatividad como «hacer algo de la nada, sino lograr algo nuevo que suele basarse en el trabajo realizado anteriormente por otros artistas, solo que siendo capaz de verlo de otra forma; las ideas van mutando y en el proceso creativo surgen nuevas formas de hacer». Explicita que «(...) los lenguajes contemporáneos, las referencias... Para mí son muy importantes». Como ejemplos: «un alumno de master de hace un par de años que utilizó referencias de 2 artistas, uno contemporáneo y otro del siglo XX, recuerdo que hizo una escultura sonora maravillosa, (...) lo llevamos a un congreso, que bautizó como "Altoparlante di ferro", (...) se basó en los móviles de Alexander Calder y también del 
artista sonoro Mikel Arce (...), hizo una escultura (...) en la que colocó material de hierro, metales, madera y un móvil perfectamente diseñado con todos los cálculos matemáticos, con sus contrapesos y [lo conectamos] a un amplificador unos micros de contacto en la barra de hierro, en lo que era la barra vertical; la base era un tronco de madera, o sea, utilizó también elementos naturales (...) la escultura funcionaba muy bien a nivel estético, (...) una estética no solo contemporánea, sino también (...) industrial, (...) que (...) visualmente funcionó muchísimo y luego a nivel sonoro resultó ser un instrumento musical porque luego con unas baquetas frotadas en la barra giraba la parte del móvil...». La obra estuvo expuesta en la Facultad de Educación. Subraya que «el estudiante era una persona que ni era músico, ni nada, era historiador que había llegado al máster, que cuando un alumno entra en este proceso creativo es capaz de hacer mucho más de lo que al principio cree que va a poder hacer» desde que asuma «el riesgo inicial, esa incertidumbre».

En su rol docente, intenta «motivarlos y casi obligarlos a transitar en esa incertidumbre. [Entonces] se logró algo espectacular; aquello no solo fue un producto artístico que funcionó muy bien, y aunque no funcione, también (...), sino que fue el resultado de un tipo de trabajo. Yo a los estudiantes les digo que, si llegamos a hacer un prototipo, ya hemos llegado muy lejos (en el poco tiempo que tenemos). $Y$ es algo muy interesante porque se ponen en marcha destrezas, habilidades y competencias diversas».

Para Elena, «la creatividad es algo importantísimo en el desarrollo de la creación musical y artística» pero destaca que sus alumnos no son músicos y disfrutan así de experiencias de apertura y entrada «en ese mundo creativo del cual espero que no salgan nunca, y sé que esto va a repercutir en los niños y niñas que caigan en sus manos, ¿no?»

De este modo de proceder «además de fomentar la creatividad personal, se logra una creatividad compartida». La clase aparece como «un lugar de encuentro», un «laboratorio de experimentación, (...) un lugar de posibilidades» que permite el desarrollo «desde el "hazlo tú mismo" hasta el "lo hacemos juntos"», en el que «A veces el error se convierte también en un motor creativo; no existe la idea buena o mala, existe la idea que atrae a otra idea...", una "explosión de ideas», de cambio y redirección, en «un camino de inicio que no sabes a donde te va a llevar, y lo importante es el viaje, ese viaje creativo».

Sus alumnos se sienten realizados con ese modo de trabajar, como constata cuando hace su evaluación. Utiliza diferentes métodos, de que destaca: individualmente, el ensayo libre en que quede patente el proceso creativo y productivo; grupalmente, se pronuncian sobre los indicadores de la evaluación; finalmente, «fomento el debate dialógico en clase, antes del proyecto, a mitad del proyecto y al final del proyecto; me siento con el grupo y digo: ¿qué pasa?, ¿qué se está "cociendo aquí"?, ¿qué elementos, qué competencias, qué habilidades, qué destrezas, a qué nivel, cómo estamos?, ¿a nivel de grupo qué pasa con las interacciones y relaciones interpersonales?» Cree que esos procesos les ayudan «a formar un autoconcepto, incluso mejorar la autoeficacia, es decir, sobre su idea de lo que son posibles de hacer a nivel creativo, y mi experiencia me dice que esto funciona».

\section{Discusión y conclusiones}

La entrevista permite a Elena discutir temas finales relativos a sus perspectivas pedagógicas.

Primero, inserta su actuación, además de en los principios del EEES, en las prescripciones de las Guías docentes de las asignaturas, definidas esencialmente a partir del trabajo de proyecto entendido holísticamente; por ejemplo: busca frecuentemente «personas fuera de la actividad del ámbito musical», que aportan visiones distintas y generadoras de inquietud 
y cambio. Registra una dura crítica refiriendo que «En la Educación Musical y Artística hay mucha labor que hacer en las escuelas, ufff, hay gente que sigue haciendo las mismas cosas, (...), y yo no digo que estén mal, pero que hay que evolucionar y hay que evolucionar un poco».

Sigue con la financiación de las actividades de los proyectos. Además del -«no mucho»«dinero asignado», los alumnos asumen por su iniciativa la compra de materiales utilizados, y Elena contribuye con su «presupuesto» personal e instrumentos/maquinaria propia.

Por fin, refiere el feed-back de los alumnos relativamente a este modo de trabajar, que destacan su satisfacción y hasta encanto con su trabajo creativo y lamentan el reducido tiempo disponible para desarrollar los proyectos.

Otro ítem referido, los problemas de relación en los grupos, que exigen que se les sensibilice para la «gestión grupal y autónoma que tiene que haber».

Indica que los alumnos confiesan que esta manera de trabajar les asusta, es complicada, surgen divergencias de opinión sobre «tanta libertad» y exigencia de autonomía debido a la larga inculcación - «fruto de una labor educativa de años, y años, y años»-, de normas y directrices relativas al qué hacer. Frente a la afirmación «si yo te doy libertad creativa no puedo decirte ;tienes que hacer esto!» frecuentemente hay «gente que es reacia (...) y esto te da para reflexionar: ¿por qué vienen así los alumnos, no?, si se habla tanto de creatividad por qué vienen pidiendo a gritos "dime lo que tengo que hacer, "dame un temario con 10 puntos que me tenga que estudiar, dime cómo vas a hacer el examen».

Por fin, relativiza el valor de las TIC, que no ve como «la panacea y la solución a la calidad educativa». En «reflexión personal», Elena subraya que no es «mejor profesora la que usa las TIC», sino que se requieren «perfiles complementarios» y sinérgicos de las competencias de los docentes, que «en todo este proceso [están] juntos»; a esta reflexión suma la constatación de la existencia de profesores poco interesados en las tecnologías pero que no por eso dejan de estar «en la línea de trabajar desde la creatividad». Fundamenta esta opinión considerando que la competencia para la creatividad es un atributo del «docente curioso, inquieto, atrevido, el inconformista, el que es capaz de asumir los riesgos de lo desconocido, el que cree que trabajar en equipo es un regalo" y añade a esas cualidades el que sea "generoso con los demás, con los colegas, que intercambia, que escucha, que detiene y cambia su mirada e intercambia ideas con los demás, que quiere descubrir nuevos caminos, explorar, transitar, aprender, aprender constantemente. $Y$ yo pienso que puede haber docentes con esas características que no utilicen la TIC; es decir, las TIC son solamente una herramienta, como un "medio para" y no un fin en sí mismas".

La entrevista permite constatar una señalada concordancia de la percepción y fundamentos pedagógicos de Elena relativamente a los elementos aportados en la fundamentación teórica y empírica del artículo; revela el potencial formativo de las TIC para el desarrollo de la creatividad, colaboración, cooperación y comunicación del profesorado en formación en materia musical, sin todavía absolutizar su valor instrumental al afirmar la importancia del fundamento de práctica pedagógica holística del profesor.

\section{Referencias}

Acosta, R., Martín-García, A. V. y Hernández, A. (2019). Uso de las Metodologías de Aprendizaje Colaborativo con TIC: Un análisis desde las creencias del profesorado. Digital Education Review, 35, 309-323. 
Alonso, E. A., Muñoz, I. y Quiñones, $M^{a}$ C. (2018). Las nuevas tecnologías en la Educación Superior. Emásf, Revista Digital de Educación Física, 55, 123-134.

Cabero, J. y Martínez, A. (2019). Las Tecnologías de la Información y Comunicación y la formación inicial de los docentes. Modelos y competencias digitales. Profesorado. Revista de currículum y formación del profesorado, 23 (3), 247-268.

Caramés, I. (2019). El uso didáctico y disciplinar de las tecnologías en la formación inicial de profesores. Didácticas Específicas, 20, 93-117.

Casanova, O. y Serrano, R. $M^{a}$ (2016). Internet, tecnología y aplicaciones para la educación musical universitaria del siglo XXI. REDU: Revista de docencia Universitaria, 14 (1), 405421.

Castañeda, L. (2019). Formación inicial del profesorado en el uso educativo de la tecnología, una propuesta curricular. Quaderns Digitals, 89, 1-49.

Castañón, $M^{a}$ R. y Vivaracho, C. E. (2012). LEEMÚSICA: Enseñanza del lenguaje musical en educación infantil (3 a 5 años) con apoyo de las TIC. Revista NEUMA, 2, 98-108.

Colás-Bravo, P. y Hernández-Portero, G. (2014). Incidencia de la Formación del Profesorado en sus creencias sobre el valor de las TIC en la enseñanza de la Música. Educatio Siglo XXI, 32 (3), 51-74.

Colás-Bravo, P. y Hernández-Portero, G. (2017). Itinerarios formativos del profesorado de Música: sus percepciones sobre el valor didáctico de las TIC. Revista Fuentes, 19 (1), 39-56.

Cózar, R., De Moya, Ma V., Hernández, J. A. y Hernández, J. R. (2015). TIC, estilos de aprendizaje y competencia musical en los estudios de Grado de Maestro. Revista Electrónica Complutense de Investigación en Educación Musical, 12, 73-85.

Díez, N. (2018). La formación en TIC de los pedagogos de música. Análisis de la situación actual en las enseñanzas superiores de música. Revista Electrónica de LEEME, 42 (2), 31-51.

Díez, N. y Carrera, X. (2018). Integración de las TIC en los procesos de enseñanzaaprendizaje de la especialidad de Pedagogía en los conservatorios superiores de música. Revista Interuniversitaria de Investigación en Tecnología Educativa (RIITE), 5, 40-55.

Escudero, J. M., Martínez-Domínguez, B. y Nieto, J. M. (2018). Las TIC en la formación continua del profesorado en el contexto español. Revista de Educación, 382, 57-80.

Espigares, M. J. y Bautista, J. M. (2018). Evaluación de objetos digitales de aprendizaje musical en Moodle. Educatio Siglo XXI, 36 (3), 377-396.

Galera, $M^{a}$ M. y Mendoza, J. (2011). Tecnología musical y creatividad: una experiencia en la formación de maestros. Revista Electrónica de LEEME, 28 (2), 24-36.

Hepp, P. (2002). Diez claves de la Educación Musical y las TICs. Educar Chile. Disponible en: http://www.educarchile.cl/ntg/docente/1556/article-75137.html

Lago, P. y Ponce de León, L. (2012). Creatividad y Tecnología en la Orientación de nuestros Futuros Músicos. Revista Iberoamericana sobre Calidad, Eficacia y Cambio en Educación, 10 (2), 136-147.

Lopes, N. y Gomes, A. (2018). Experimentar con TIC en la formación inicial de profesores. Educatio Siglo XXI, 36 (3), 255-274. 
Lores, B., Sánchez, P. y García, Ma R. (2019). La formación de la competencia digital en los docentes. Profesorado. Revista de currículum y formación del profesorado, 24 (2), 234-260.

Marqués, G. (2004). Metodología Didáctica y TIC en la enseñanza universitaria. Disponible en: http://dewey.uab.es/PMARQUES/caceres.html

Martín-Piñol, C., Calderón-Garrido, D. y Gustems-Carnicer, J. (2016). Interdisciplinariedad y tecnología en la educación artística desde la experiencia creativa. Arte y políticas de identidad, 14, 79-95.

Padula, J. E. (2016). La mediación creativa. El autor y el compositor de música en el mundo de las TIC. Revista Mexicana de Comunicación, 1 (139), 25-29.

Palau, R., Usart, M. y Ucar, Ma J. (2019). La competencia digital de los docentes de los conservatorios. Estudio de autopercepción en España. Revista Electrónica de LEEME, 44 (2), 24-41.

Palazón-Herrera, J. (2016). Una aproximación al uso y competencia tecnológica del profesorado de música de Educación Secundaria. En J. L. Castejón (Coord.), Psicología y educación: Presente y futuro (pp. 885-892). Madrid: Asociación Científica de Psicología y Educación (ACIPE).

Rodríguez, Z. J. y Guzmán de Castro, B. (2012). Competencias en el uso de las TIC en profesores de la especialidad de educación musical del Instituto Pedagógico José Manuel Siso Martínez. Revista de Investigación, 76 (36), 35-55.

Roldán, G. J. (2016). Educación musical de adultos en la Universidad de Granada y alfabetización digital. Opción, 12, 460-475.

Torres, L. C. (2017). Creatividad artificial. Revista de Tecnología, 16 (2), 18-26.

Touriñán, L. (2018). Música, educación y nuevas tecnologías: fundamentos pedagógicos de la relación. Educación "por" la música en la formación adulta universitaria a través de las TIC. Boletín Redipe, 7 (7), 39-77.

Vaquer, A. y Vera-Muñoz, $M^{a}$ I. (2012). El profesorado de música y el uso del software libre. Revista Estudios, 25, 311-326.

Vargas, E., Gértrudix, F. y Gértrudix, M. (2015). Los procesos colaborativos de la composición musical on line. El caso de la plataforma "poliedro". Revista Electrónica de Investigación, Docencia y Creatividad, 4, 20-37.

Vecina, Ma L. (2006). Creatividad. Papeles del psicólogo, 27 (1), 31-39. 